\title{
Medial Frontal Cortex in Action Monitoring
}

\author{
Phan Luu, ${ }^{1,2}$ Tobias Flaisch, ${ }^{3}$ and Don M. Tucker ${ }^{1,2}$

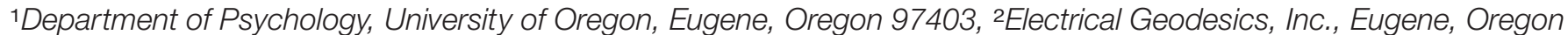 \\ 97403 and 3 University of Konstanz, 78457 Konstanz, Germany
}

\begin{abstract}
Effective behavior requires continuous action monitoring. Electrophysiological studies in both monkeys and humans have shown activity in the medial frontal cortex that reflects dynamic control and monitoring of behavioral acts. In humans, the centromedial frontal cortex shows an electrical response within 100 msec of an error, the error-related negativity (ERN). The ERN occurs only when subjects are aware of making an error, suggesting that a critical factor may be self-monitoring of the action process. In the present study, we examined late responses in a deadline reaction time task, in which the subject becomes increasingly aware of making an error as the response
\end{abstract}

becomes increasingly late. We found evidence of response conflict before errors defined by late responses but not before errors defined by incorrect responses. The results also show a linear increase in the amplitude of the ERN with increasingly late responses. These data suggest that frontal networks provide dynamic representations that monitor and evaluate the unfolding action plan.

Key words: anterior cingulate; error-related negativity; action monitoring; awareness; response conflict; event-related potential
Action monitoring is an important component of self-regulation. This component is best appreciated when self-monitoring is defective, as in patients with the alien-hand syndrome. With lesions to the centromedial aspect of the prefrontal lobe, including the supplementary motor area (SMA), these patients feel as if certain self-initiated actions have a foreign source, i.e., as if some of their actions are not volitional (Goldberg, 1985). In recent years, scalp electrophysiological studies in humans have provided insight into the neurophysiological processes of action monitoring and error detection. Falkenstein et al. (1991) identified a negative deflection in the event-related potential (ERP) that is associated with an error response [the $\mathrm{N}_{\mathrm{E}}$ or the $\mathrm{N} 2 \mathrm{c}$ by Kopp et al. (1996)]. This negativity can be observed in both stimulus-locked and responselocked ERPs. It has a frontocentral distribution and peaks within 80-110 msec after the response in response-locked averages. Other researchers independently observed this effect (Gehring et al., 1993) and termed it the error-related negativity (ERN). Dipole modeling of a 64-channel recording showed that the neural source of the ERN lies in the vicinity of the anterior cingulate gyrus (Dehaene et al., 1994). However, because of inaccuracies of dipole models, contributions from other areas of the medial prefrontal cortex, including the SMA, cannot be ruled out.

The amplitude of the ERN does not seem to be influenced by stimulus differences (Bernstein et al., 1995), nor does it seem to be involved in attempts to inhibit or correct erroneous responses (Scheffers et al., 1996) or in making response selections (Badgaiyan and Posner, 1998). It can be observed in error responses during both auditory and visual tasks (Falkenstein et al., 1991). Although error feedback can be sufficient (Miltner et al.,

\footnotetext{
Received July 21, 1999; revised Oct. 12, 1999; accepted Oct. 14, 1999.

This research was supported by the National Institute of Mental Health Grants MH42129 (Depression and Anxiety as Neural Control Processes) and MH42669 (Depression and Spatial Orienting) to D.M.T. We thank Michael Posner for his careful reviews.

Correspondence should be addressed to Dr. Phan Luu, Department of Psychology, University of Oregon, Eugene, OR 97403. E-mail address: pluu@oregon. uoregon.edu.

Copyright (C) 1999 Society for Neuroscience $\quad 0270-6474 / 99 / 200464-06 \$ 15.00 / 0$
}

1998), it is not required to produce the ERN (Gehring et al., 1993).

In the anterior cingulate cortex, single-cell recordings in monkeys have revealed cells that track the commission of an error (Niki and Watanabe, 1979). Importantly, these same cells also respond to the omission of a reward for a correct response. Motivational factors also influence the amplitude of the ERN in human studies. Tucker et al. (1999) observed a centromedial frontal negativity (stimulus-locked, at $\sim 480 \mathrm{msec}$ ) in response to task feedback, with a more negative deflection for negative than for positive feedback. This effect was larger for subjects who reported either pleasant or unpleasant arousal during the task, suggesting that it may have reflected the subject's level of motivation.

Gehring et al. (1993) originally observed that the amplitude of the ERN was larger when task instructions emphasized accuracy rather than speed of responding. More recently, Gehring et al. (2000) showed that the amplitude of the ERN was larger in obsessive-compulsive patients than in normal controls, and within the patients it was larger for those with more severe symptoms. Luu et al. (2000) also found that in the initial stages of the experiment high-negative affect subjects (characterized as subjects who experience high levels of subjective distress) initially exhibited larger ERN amplitudes compared with the low-negative affect subjects. Dikman and Allen (2000) found that individuals with low-trait socialization exhibit smaller ERN amplitudes in tasks that penalize error responses when compared with hightrait socialization individuals. No differences in ERN amplitude were observed between these two groups when error responses prevented the earning of rewards. Thus, subjects who are characterized as being high or low on the dimension of subjective distress and who engage in excessive self-monitoring show exaggerated ERNs.

Despite the increasing amount of evidence from these experiments, the psychological nature of the ERN remains unclear. Some authors have suggested that it indexes error-related moni- 
toring (Gehring et al., 1993), whereas others have argued that it indexes response conflict (Carter et al., 1998). More recently, Carter et al. (1999) have emphasized an evaluative role for the anterior cingulate. In this model, the anterior cingulate specifically evaluates (i.e., detects) processing conflicts that may lead to a decrement in behavioral performance. Because of the centromedial frontal distribution of the ERN, a response-conflict interpretation is consistent with the many findings of anterior cingulate activity under conditions of both cognitive and response conflict (Pardo et al., 1990; Posner and DiGirolamo, 1998). Another important issue is whether awareness is required to generate the ERN. Dehaene et al. (1994) showed that when subjects were unaware of making an error, no ERN was observed.

In a speeded task, subjects can commit two types of errors. The first type of error is the error of commission. This type of error is a response hand error. Given a particular response deadline, not all correct responses will occur before the imposed time limit. These late responses make up the second class of errors: errors of speed. Both types of error responses (incorrect responses and errors of speed) are defined as erroneous by the parameters of the task because points are deducted in both cases. In the present study, errors of speed are similar to the errors defined in time estimation tasks used by Miltner et al. (1998) and Badgaiyan and Posner (1998). In other words, in these two studies, responses must be generated within a particular time interval for them to qualify as correct. What these researchers find are that errors in these tasks are not obvious to subjects and that an error feedback signal is required to generate the ERN.

Errors of speed are particularly interesting. As a response becomes increasingly late, subjects become increasingly aware that the response is past the deadline and thus in error. Explicit awareness of the error may not be the critical factor, of course. Significant increases in self-monitoring may be underway before subjects are able to report that they are aware of being late.

In the present study, we sorted responses in a speeded task into those that were slightly, moderately, and very late. To examine the role of response conflict as subjects were attempting to decide these late responses, we analyzed the lateralized readiness potential (LRP). The first hypothesis was that greater response conflict as evidenced by the LRP would be associated with greater motor preparation of the incorrect response and that this index of response conflict would correlate with the magnitude of the ERN. The second hypothesis was that increasing lateness would be associated with increasing self-monitoring and with increasing amplitude of the ERN, even though the response was the correct choice.

\section{MATERIALS AND METHODS}

Subjects. Twenty-one subjects participated in the experiment. Subjects were recruited from the Department of Psychology's subject pool. All subjects participated to fulfill a course requirement. Subjects also had opportunities to earn a monetary reward as described in Procedure. All subjects had normal or corrected-to-normal vision and were fluent in English. They ranged in age from 18 to 26 years $(19.4 \pm 1.9$, mean \pm SD). Eight subjects were male, and 19 subjects were right-handed. None of the subjects was currently taking prescription medications. These subjects are a random subset of the subjects reported in Luu et al. (2000).

Task. The task used is known as the Eriksen flanker task (Eriksen and Eriksen, 1979). It was used originally to study stages of information processing. Important for our purposes, this task also requires subjects to monitor their responses as they make speeded decisions. It has been used by Gehring et al. (1993) to study the ERN. In this task, subjects were presented with a warning cue, an asterisk, at the center of a screen above a fixation mark, which was present for the entire experiment. The warning cue was presented for $804 \mathrm{msec}$, after which it was replaced with one of the following strings of target letters: HHHHH, SSHSS, SSSSS, or HHSHH. The bottom of the letter strings was presented $0.1^{\circ}$ above the fixation mark, and each letter subtended $0.5^{\circ}$. The probability of each string of letters was 0.25 . The target letter string was presented for $107 \mathrm{msec}$.

The subject's task was to indicate as quickly and as accurately as possible which letter was in the center by pressing a key with one hand if the center letter of the target string was an $\mathrm{H}$ and another key with the opposite hand if the center letter was an S (keys were counterbalanced across subjects). The timing of the reaction time (RT) began with the presentation of the target.

The criterion for RT deadline for each subject was based on his or her median RT on practice trials. During the practice trials, subjects were informed that the deadline was set at $400 \mathrm{msec}$. Subjects were presented with practice trials in blocks of 40 . The median RT was only determined for the last 30 trials of each block, because the first 10 trials were used to orient the subjects to the task. Subjects were allowed to practice as much as they wanted. However, most subjects only chose to complete one block of practice trials (40). Subjects were not informed that their median RT from the practice trials would be used to determine their RT deadline for the task; however, they were instructed regarding the new deadline value that would be used during the formal task run that followed. The range of the RT deadline across subjects, as determined from the practice trials, was $343-651 \mathrm{msec}(408 \pm 96 \mathrm{msec}$, mean $\pm \mathrm{SD})$. The response interval was $1005 \mathrm{msec}$ from the time of target onset. If subjects did not respond by this time for a particular trial, no response time was recorded, and the trial was marked as having no response.

After the response interval, a feedback signal informing the subject of the status of the response was presented above the fixation mark. The feedback could indicate that the response was correct and on time (a plus sign), correct but too late (an exclamation point), or incorrect or no response (a minus sign). The feedback signal was presented for 1099 msec. The recording of the EEG began $201 \mathrm{msec}$ before cue onset and terminated with the removal of the feedback signal. The intertrial interval varied between 1525 and $2505 \mathrm{msec}$.

EEG recording. EEG was recorded from 128 scalp sites using the 128-channel Geodesic Sensor Net (Tucker, 1993). The impedance of all electrodes was between 10 and $40 \mathrm{k} \Omega$. All recordings were initially referenced to $\mathrm{Cz}$. EEG was recorded using a $0.1-50 \mathrm{~Hz}$ bandpass $(3 \mathrm{~dB}$ attenuation). The signals were sampled at $125 \mathrm{samples} / \mathrm{sec}$ and were digitized with a 12-bit analog-to-digital converter.

Because of volume conduction, no site on the head, including traditional reference sites such as the mastoids or earlobes, can be regarded as being an "inactive" reference site (Tucker et al., 1994). Therefore the EEG was rereferenced against an average reference (Bertrand et al., 1985). Editing of the EEG for movement, eye movement, blink artifacts, and noise was performed by a computer algorithm. The artifact-free EEG was then averaged locked to the response for each subject according to the different types of responses: correct, Early late, Mid late, Late late, and error.

EEG averaging. Correct responses were those responses that consisted of the correct hand and occurred before the deadline. Error responses were defined as responses with the incorrect hand, regardless of speed. Late responses were defined as responses with the correct response hand but whose latency exceeded the response deadline. Because we are interested in response conflict, only incompatible trials (i.e., HHSHH and SSHSS) were included in the averages and in the analyses of the late responses (for both the lateralized readiness potential and late ERN measures). Late responses were separated into three categories according to the following procedure: For each individual subject, his or her late responses were rank-ordered and divided into three even intervals (i.e., bins). The intervals were labeled Early late, Mid late, and Late late. The Early late interval contained responses that barely exceeded the RT deadline, and the Late late interval contained responses that were the latest, with the Mid late interval in between. The RT values for each of these intervals were computed separately for each subject.

After the EEG was averaged according to the five different response types, it was baseline-corrected using the $400 \mathrm{msec}$ window before the response and digitally filtered with a $30 \mathrm{~Hz}$ low-pass filter (using a 600-400 msec preresponse baseline did not affect the results).

Lateralized readiness potential. The bereitschaftspotential (BP) or motor readiness potential is a negative-going deflection in the EEG centered around the dorsal motor and mediodorsal areas before a motor response. It is believed to reflect the preparatory set and intention to act (Lang et al., 1988). This potential can begin up to a second or more 
before a self-paced act. When the BP is taken as a difference between the contralateral and ipsilateral sites, it is referred to as the LRP and is used as a measure of motor priming by stimulus events (see Coles et al., 1988). By using the LRP, we can measure response competition directly.

Procedure. After being prepared for the EEG recording by application of the Geodesic Sensor Net (Tucker, 1993), subjects were seated in front of a monitor in an EEG recording booth, with distance and alignment to the screen controlled by use of a chin rest. After completion of recording of the resting EEG, subjects were given instructions on how to perform the task.

Each subject started the experiment with 3200 points. Subjects were informed that if they responded correctly and before a predetermined time limit no points would be lost. Subjects were also told that correct but late responses (errors of speed) were penalized 1 point for every 100 msec that they were tardy and that response errors (errors of commission) would result in a loss of 8 points. To increase motivation to perform well on the task, we told the subjects that the total amount of points that they managed to retain by the end of the task would be rewarded at a rate of 0.5 cent for every point. It was emphasized that this monetary reward was in addition to the course credits that they would receive for participating.

As a further manipulation of motivation levels, subjects were informed that at the end of the study they would be given feedback about their overall performance relative to others who had performed the task. (All subjects exerted adequate effort on the task and were told that they had done well.) Subjects then proceeded to practice the task. After subjects were comfortable with the task, they completed 800 trials, broken down into 200-trial blocks. Between blocks, subjects were allowed to rest and move about. The time spent on the task took between 1 and $1.5 \mathrm{hr}$.

\section{RESULTS}

\section{Behavioral data}

First, we analyzed the distribution of stimulus type (compatible vs incompatible) according to late responses. As expected, lateness of response interacted with stimulus compatibility $\left[F_{(2,40)}=\right.$ $12.4 ; p<0.001]$. This interaction indicated that more incompatible trials were associated with the Late late than with the Early late responses and conversely that fewer compatible trials were associated with the Late late than with the Early late responses. All subsequent analyses that include late responses will only contain data from incompatible trials, because we are specifically interested in response competition.

The median RT of each late interval was obtained and entered into an ANOVA model with response type (correct, Early late, Mid late, Late late, and error) as the only factor. The analysis revealed that there was a significant difference in median RT between response types $\left[F_{(4,80)}=227.3\right.$; Geisser-Greenhouse corrected $p<0.001]$. Means comparisons revealed that the correct and error responses did not differ in median reaction time. In contrast, the correct RTs were significantly faster than Late late RTs $\left[F_{(1,80)}=646.4 ; p<0.001\right]$. These results are not surprising because the reaction times themselves were the basis of responsetype separation for the late responses.

\section{Response-locked ERP data}

\section{Lateralized readiness potential}

In the present study, we averaged the EEG to correct, Early late, Mid late, Late late, and error responses separately for left- and right-hand responses. The LRP was then defined for each response type as the average of the difference between the EEG over the contralateral motor cortex (to the response hand) minus the EEG over the ipsilateral motor cortex. In other words, the LRP is defined as: $\left[\left(\mathrm{C}^{\prime}-\mathrm{C}^{\prime}\right)+\left(\mathrm{C}^{\prime}-\mathrm{C} 4^{\prime}\right)\right] / 2$. Within this formula, the average should be zero, or close to zero, when there is no (or equal) activity observed over the motor cortex. However, as motor cortices are activated asymmetrically according to the response hand, the value departs from zero. Because appropriate

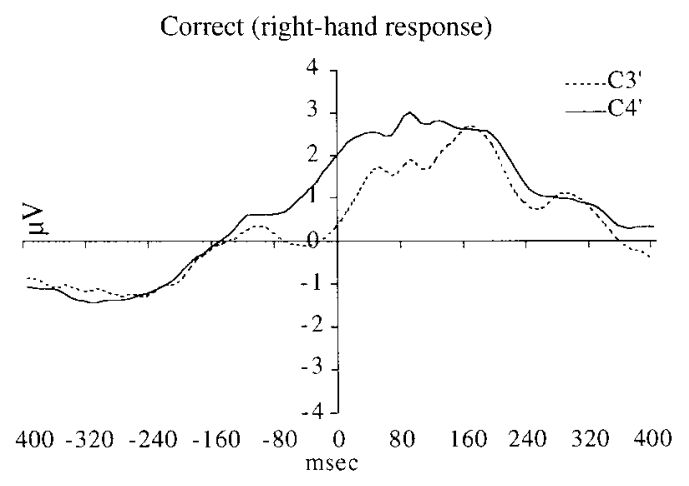

Late late (right-hand response) Incompatible

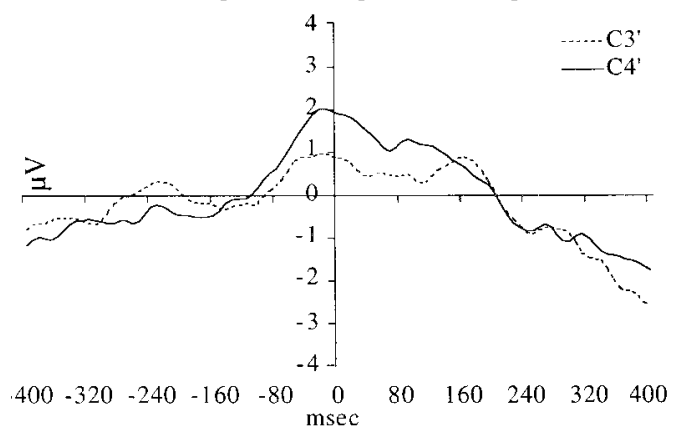

Error (right-hand response)

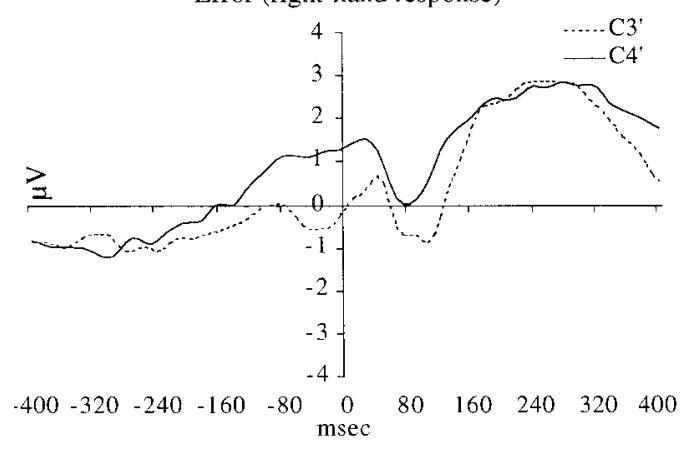

Figure 1. Raw voltage waveforms at $\mathrm{C}^{\prime}$ and $\mathrm{C}^{\prime}$ ' for three representative conditions. Right-hand key presses for correct (top), Late late (middle), and error (bottom) responses. Time 0 marks the time of response.

motor priming is observed over the contralateral motor cortex as a developing negativity before a response, correct motor priming should be observed as a negative deflection in the LRP according to the above formula.

Because the LRP is a difference wave, interpretation of the difference should rely on careful inspection of the original waveforms to determine the source of the difference. Figure 1 presents the raw voltage waveforms for three representative conditions. It can clearly be seen that when subjects make a correct right-hand response there is minimal difference between the electrodes overlying the motor cortex. In contrast, when subjects make a righthand response that is late, the voltage over the ipsilateral motor area is more negative. Conversely, when subjects respond with the right hand and it is an error, the contralateral motor area is more negative. Thus, in the present study the LRP over all the conditions (Fig. 2) does appropriately reflect asymmetric motor activation.

It can be observed in Figure 2 that $\sim 300 \mathrm{msec}$ before response onset, the LRPs for the five response types begin to diverge from each other. The amplitudes of the LRPs associated with correct 


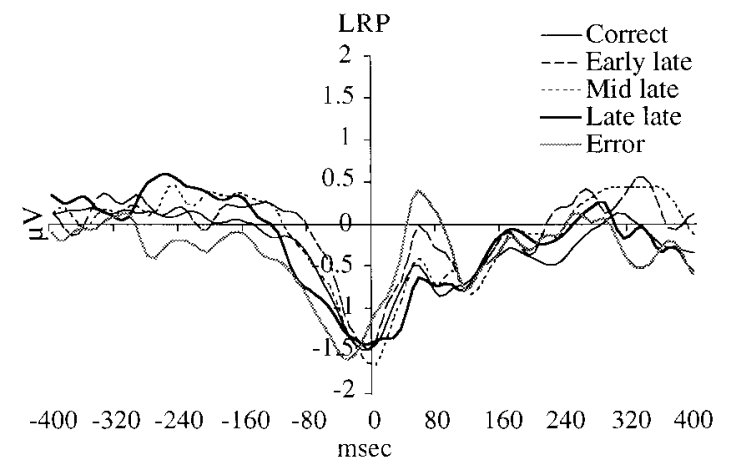

Figure 2. The lateralized readiness potentials for the five response types. Time 0 marks the time of response.

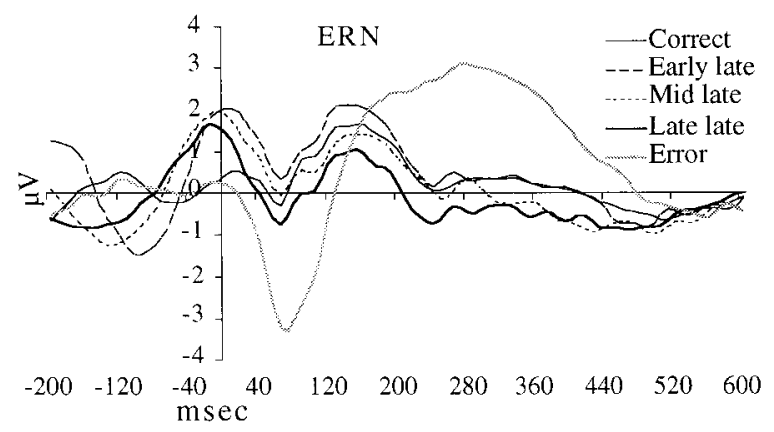

Figure 3. The error-related negativity, shown at a channel $\sim 3 \mathrm{~cm}$ rostral to $\mathrm{Cz}$, can be observed in all types of responses, but its amplitude is largest for error responses. The time of response is marked by the vertical line.

and Early late responses are smallest. Next, in order of positive LRP amplitudes, are the Mid late and Late late responses. These LRPs indicate that the later the response, the more likely it was that subjects were preparing to respond with the incorrect hand. The error responses, in contrast, are associated with a negative deflection in the LRP, indicating that there was no ambivalence, and only preparation of the motor cortex contralateral to the hand that actually responded (which was in this case the wrong response).

We took the average amplitude of the LRP between 280 and $160 \mathrm{msec}$ before response onset for all five types of responses. These values were entered into an ANOVA model with response type as the single factor. The analysis revealed a significant effect of response type $\left[F_{(4,80)}=7.7 ; \mathrm{G}-\mathrm{G} p<0.001\right]$. A means comparison confirmed the above differences between the correct and Late late responses $\left[F_{(1,80)}=9.1 ; p<0.01\right]$ and the error and Late late responses $\left[F_{(1,80)}=26.6 ; p<0.001\right]$.

\section{Error-related negativity}

Figure 3 shows the response-locked ERP for each type of response at the centromedial frontal ERN maximum site (channel $6, \sim 3 \mathrm{~cm}$ rostral to $\mathrm{Cz}$ ). The ERN can be clearly observed for error responses, peaking $\sim 80 \mathrm{msec}$ after response onset. However, ERNs also can be seen for late responses (errors of speed but not of action). Moreover, the amplitude of the ERN varies linearly with the lateness of the response.

To verify that the late ERNs are similar to the ERN observed for error responses, topographic maps of the correct, late (collapsed across all three late types), and error responses are displayed in Figure 4. In Figure 4 the data were filtered with a $4 \mathrm{~Hz}$

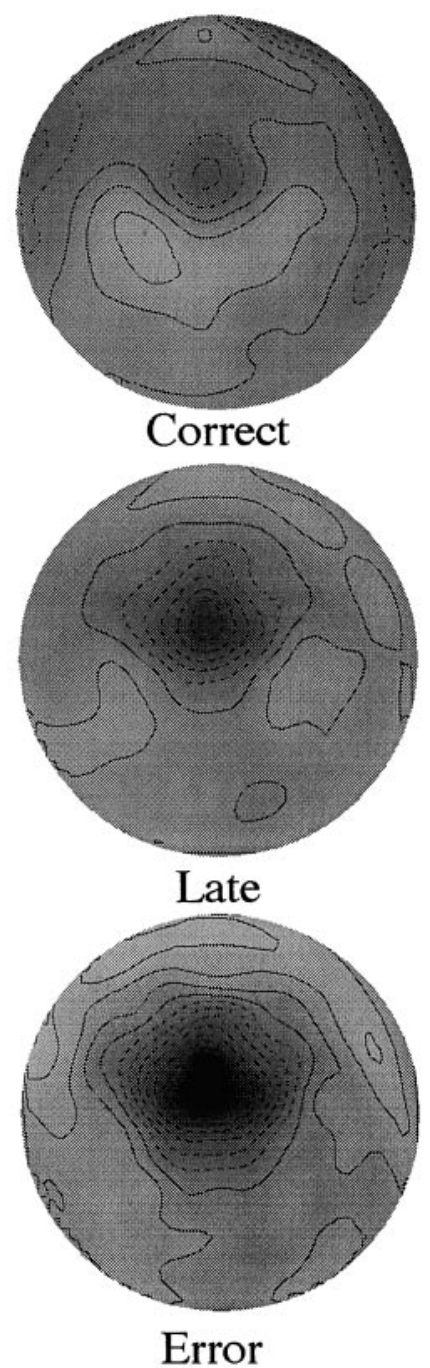

Figure 4. Topographic map of the error-related negativity at $80 \mathrm{msec}$ after response for correct, late, and error responses. Darker colors indicate greater negativity. The orientation of the map is top looking down and nose toward the front. See Results for details.

high-pass filter to remove the slow, parietal positivity associated with response onset. This parietal positivity possibly reflects the P3 associated with the stimulus, because most reaction times fall within the P3 interval (Luu et al., 2000). Removing the large positivity that overlaps with the ERN allows for the grayscale palette to cover the range of the ERNs associated with the correct and late responses (which are of much smaller amplitude) and thus to bring out the topography of the negativity. Figure 4 shows that the negativity associated with the correct and late responses has the same topographic distribution as that of the ERN for error responses.

We measured the average potential of the ERN over a 40-144 msec postresponse epoch from channel 6 for the five response types and entered the values into an ANOVA model. Response type was entered as the single factor into the model. The results revealed a significant main effect $\left[F_{(4,80)}=10.6 ; \mathrm{G}-\mathrm{G} p<0.001\right]$. Means comparisons confirm that the ERP associated with the Late late response type was significantly less positive than the ERP associated with the Early late response type $\left[F_{(1,80)}=6.0\right.$; $p<0.04]$. The error response type was significantly more nega- 
tive than all of the other types $\left[F_{(1,80)}=22.7 ; p<0.001\right.$ (correct); $F_{(1,80)}=36.1 ; p<0.001$ (Early late); $F_{(1,80)}=22.3 ; p<0.001$ (Mid late); $F_{(1,80)}=12.6 ; p<0.005$ (Late late)]. We also tested whether there were latency differences between the different response types but found no significant differences.

\section{DISCUSSION}

In this study, we examined human frontal lobe electrical activity in relation to errors of inaction (taking too long to respond) as well as errors of incorrect action (pressing the wrong button). ERNs over the centromedial frontal lobe were seen within 100 msec of the response in both cases. In this speeded task, subjects knew that they had to respond within a target interval or the response would be considered late and scored as an error. The late responses varied from those barely missing the deadline to ones that were made much later. We hypothesized that as responses become increasingly late, the self-monitoring of these responses should become increasingly strong and the ERNs should increase linearly. By comparing the ERP associated with different degrees of response lateness, we tested this hypothesis and confirmed that increasingly late responses were associated with increasingly large ERNs.

We interpret these findings to indicate that self-monitoring is integral to the ERN. For an early response, there are only moderate demands on self-monitoring. For an increasingly late response, as subjects try to avoid the error of responding past the deadline, there are increasing demands for attentional selfmonitoring that appear to be reflected in increasing activity in the centromedial frontal cortex.

Analysis of the LRP suggested that the late responses were also associated with response conflict. The LRP is a measure of asymmetric motor preparation, and it has been used as a measure of motor preparation in response to task demands (Coles et al., 1988; Hackley and Valle-Inclan, 1998). The LRP has also been used to study the effects of response competition arising from incongruent primes that are presented subconsciously (Dehaene et al., 1998). Dehaene et al. (1998) found that the response competition indexed by the LRP can be validated using functional magnetic resonance-imaging (fMRI) methods. These researchers used a lateralized measure of the BOLD response in motor cortices and found the effect to be identical to the effect using the LRP (i.e., incongruent primes activated the wrong motor areas). Finally, the LRP has been used to study early response activation in classical conflict paradigms, such as the Eriksen flanker task and the Stroop task, and to study incompatible response priming induced by incompatible noise (Coles et al., 1988).

In the present study, the late responses were associated with inappropriate priming of the motor cortex ipsilateral to the eventual response hand. By looking at just the incompatible trials, it could be seen that the degree of response lateness is related to the degree of priming of the inappropriate response hand. The LRP that is observed is likely caused by the effects of the incompatible stimuli, because the onset of the LRP occurs $\sim 100$ msec after stimulus onset. That is, the asymmetric motor priming begins $\sim 300 \mathrm{msec}$ before the response (the responses range between 300 and $700 \mathrm{msec}$ after stimulus onset).

Although increasing response conflict was associated with the increased ERN for late responses, there was no LRP evidence of response competition for the incorrect response errors. Rather, the subjects appeared to make the wrong response without any (conflicted) preparation of the correct response. Because the LRP is a difference measure, it is possible that high levels of conflict (i.e., high levels of simultaneous activation of both left and right motor cortices) can result in near-zero LRP values. Figure 1 shows, however, that the condition in which equal activity is observed over the motor cortices is the correct response condition. For responses in which the most response conflict is expected (i.e., Late late and error responses), the motor activation is maximally asymmetric. The wrong hand is primed in both cases. For the Late late responses, the motor activation eventually switches to the side contralateral to the correct hand. For the error responses, it remains with the wrong hand, and the wrong response key is pressed. Although Figure 1 shows the data for the right hand, identical effects were observed for the left-hand responses. Therefore, we conclude from the present data that response conflict was associated with the ERN for one kind of error in this experiment (the late responses) but not another (the incorrect responses).

There was a brief inversion of the LRP after the response was made (Fig. 2 at $\sim 60 \mathrm{msec}$ after the response), and this effect was greater for incorrect response errors than for late errors. Thus, judging from the LRP, one might think that a brief period of response conflict, or perhaps response correction (activating the motor area for the correct hand), could have occurred immediately after the incorrect response (at $\sim 60 \mathrm{msec}$ after the response), just before the ERN (at $\sim 80 \mathrm{msec}$ after the response). However, inspection of the waveforms from both left- and righthand errors shows that this postresponse inversion of the LRP was caused by a brief negative deflection over the contralateral motor cortex $\left(\mathrm{C}^{\prime}\right)$ of the correct, nonresponding, right hand only for the left-hand error response. It would be expected that if this was an index of a response correction, then this negativity would be observed over C4' (contralateral to the correct, nonresponding hand) for the right-hand errors, but in fact, the pattern was the same as that of the left-hand errors. In debriefing, subjects in fact reported that they experienced little or no response conflict on the incorrect response trials. Several subjects reported that the error responses felt impulsive and that they had to slow down deliberately to avoid committing consecutive errors.

According to the response-conflict monitoring hypothesis proposed by Carter et al. (1998), error responses are made under conditions of high response conflict. Although this explanation fits well with the late responses of the present study, it does not fit the data from incorrect responses. The response-conflict hypothesis also has difficulty explaining the studies using error feedback to elicit the ERN (Badgaiyan and Posner, 1998; Miltner et al., 1998). It was argued by Badgaiyan and Posner that error feedback does not require a generation of a response, and therefore, the ERN is not associated with motor or premotor events. This suggestion has been confirmed recently by Leuthold and Sommer (1999) using response-locked ERNs. These researchers were able to show that the ERN is not part of the stimulus-response pathway. Rather, they argue that the ERN reflects a general error-processing mechanism.

Although the response-conflict hypothesis remains an important one for explaining anterior cingulate and medial frontal activation during errors, we favor an interpretation that emphasizes the more general function of self-monitoring of the motor plan. Conflicting responses inherently engage self-monitoring, but self-monitoring often occurs in the absence of conflicting responses.

Results from animal studies imply that self-monitoring of the error is required for the appearance of error-related cortical potentials (Brooks, 1986; Gemba et al., 1986). Brooks et al. 
observed cortical potentials in monkeys that were related to errors in task performance. These potentials were similar to the human ERN in several ways. First, they were recorded in the anterior cingulate cortex. Second, they were surface-negative. Finally, the time course was very similar to that of the ERN. Importantly, before the monkey had gained an effective representation of the correct response for the task, the error responses did not elicit error potentials. Thus, the current awareness of the difference between correct and error responses (or at least the working memory substrate of monkey awareness) appears essential to the formation of error-related potentials.

The present results provide further evidence that activity in the medial frontal lobe closely parallels the control of attention (Posner and Rothbart, 1998). Because ERN-like centromedial negativities are seen in stimulus-locked ERPs when subjects discriminate good from bad targets in a video game (Tucker et al., 1999), or in other experiments when a feedback signal is given (Miltner et al., 1998), motivational evaluation appears integral to this class of frontal lobe responses. Similarly, when subjects make decisions on whether emotionally significant trait words (e.g., stingy, bold, or geek) apply to them, a centromedial effect at $350 \mathrm{msec}$ after the stimulus discriminates both endorsement and the emotional valence (good/bad) of the words (D. M. Tucker, A. Hartry-Speiser, R. Desmond, L. McDougal, T. Flaisch, and P. Luu, unpublished observations). These reports are consistent with the well known effects of medial prefrontal lesions, which cause patients to be apathetic and unconcerned about the consequences of their actions (Rylander, 1947; Tucker et al., 1995). Considering the evidence in its entirety, we propose that the core contribution to attention from the anterior cingulate gyrus and associated medial frontal cortex is evaluative self-monitoring along an affective dimension. When subjects are concerned with the outcome of an action, the cingulate gyrus and associated centromedial frontal lobe provide a dynamic monitoring of that action and its effects. As evidenced in the present findings of progressively larger ERNs to responses increasingly past the deadline, this self-monitoring process appears to be a dynamic operation, providing continuous motivational evaluation, and presumably continuous executive guidance, to direct the neural mechanisms of behavior.

\section{REFERENCES}

Badgaiyan RD, Posner MI (1998) Mapping the cingulate cortex in response selection and monitoring. NeuroImage 7:255-260.

Bernstein PS, Scheffers MK, Coles GH (1995) "Where did I go wrong?" A psychophysiological analysis of error detection. J Exp Psychol 21:1312-1322.

Bertrand O, Perrin F, Pernier J (1985) A theoretical justification of the average-reference in topographic evoked potential studies. Electroencephalogr Clin Neurophysiol 62:462-464.

Brooks VB (1986) How does the limbic system assist motor learning? A limbic comparator hypothesis. Brain Behav Evol 29:29-53.

Carter CS, Braver TS, Barch DM, Botvinick MM, Noll D, Cohen JD (1998) Anterior cingulate cortex, error detection, and the online monitoring of performance. Science 280:747-749.

Carter CS, Botvinick MM, Cohen JD (1999) The contribution of the anterior cingulate cortex to executive processes in cognition. Rev Neurosci 10:49-57.

Coles MGH, Gratton G, Donchin E (1988) Detecting early communication: using measures of movement-related potentials to illuminate human information processing. Biol Psychol 26:69-89.

Dehaene S, Posner MI, Tucker DM (1994) Localization of a neural system for error detection and compensation. Psychol Sci 5:303-305.
Dehaene S, Naccache L, Le Clec'H G, Koechlin E, Mueller M, DehaeneLambertz G, van de Moortele PF, Le Bihan D (1998) Imaging unconscious semantic priming. Nature 395:597-600.

Dikman ZV, Allen JJB (2000) Error monitoring during reward and avoidance learning in high and low-socialized individuals. Psychophysiology, in press.

Eriksen CW, Eriksen BA (1979) Target redundancy in visual search: do repetitions of the target within the display impair processing? Percept Psychophys 26:195-205.

Falkenstein M, Hohnsbein J, Hoormann J, Blanke L (1991) Effects of crossmodal divided attention on late ERP components. II. Error processing in choice reaction tasks. Electroencephalogr Clin Neurophysiol 78:447-455.

Gehring WJ, Goss B, Coles MGH, Meyer DE, Donchin E (1993) A neural system for error detection and compensation. Psychol Sci $4: 385-390$.

Gehring WJ, Himle J, Nisenson LG (2000) Action monitoring dysfunction in obsessive-compulsive disorder. Psychol Sci, in press.

Gemba H, Sasaki K, Brooks VB (1986) "Error" potentials in limbic cortex (anterior cingulate area 24) of monkeys during motor learning. Neurosci Lett 70:223-227.

Goldberg G (1985) Supplementary motor area structure and function: review and hypotheses. Behav Brain Sci 8:567-615.

Hackley SA, Valle-Inclan F (1998) Automatic alerting does not speed late motoric processes in a reaction-time task. Nature 391:786-788.

Kopp B, Rist F, Mattler U (1996) N200 in the flanker task as a neurobehavioral tool for investigating executive control. Psychophysiology 33:282-294

Lang W, Lang M, Uhl F, Koska C, Kornhuber A, Deecke L (1988) Negative cortical DC shifts preceding and accompanying simultaneous and sequential finger movements. Exp Brain Res 71:579-587.

Leuthold H, Sommer W (1999) ERP correlates of error processing in spatial S-R compatibility tasks. Clin Neurophysiol 110:342-357.

Luu P, Collins P, Tucker DM (2000) Mood, personality, and self-monitoring: negative affect and emotionality in relation to frontal lobe mechanisms of error-detection. J Exp Psychol Gen, in press.

Miltner WHR, Braun CH, Coles MGH (1997) Event-related brain potentials following incorrect feedback in a time-estimation task: evidence for a "generic" neural system for error detection. J Cogn Neurosci 9:788-798.

Niki H, Watanabe M (1979) Prefrontal and cingulate unit activity during timing behavior in the monkey. Brain Res 171:213-224.

Pardo JV, Pardo PJ, Janer KW, Raichle ME (1990) The anterior cingulate cortex mediates processing selection in the stroop attentional conflict paradigm. Proc Acad Sci USA 87:256-259.

Posner MI, DiGirolamo G (1998) Executive attention: conflict, target detection and cognitive control. In: The attentive brain (Parasuraman R, ed), pp 401-423. Cambridge, MA: MIT.

Posner MI, Rothbart MK (1998) Attention, self regulation and consciousness. Philos Trans R Soc Lond [Biol] 353:1-13.

Rylander G (1947) Personality analysis before and after frontal lobotomy. In: Research publications association for research in nervous and mental disease: the frontal lobes (Fulton JF, Aring CD, Wortis BS, eds), pp 691-705. Baltimore: Williams \& Wilkins.

Scheffers MK, Coles MGH, Bernstein P, Gehring WR, Donchin E (1996) Event-related brain potentials and error-related processing: an analysis of incorrect responses to go and no-go stimuli. Psychophysiology 33:42-53.

Tucker DM (1993) Spatial sampling of head electrical fields: the geodesic sensor net. Electroencephalogr Clin Neurophysiol 87:145-163.

Tucker DM, Liotti M, Potts GF, Russell GS, Posner MI (1994) Spatiotemporal analysis of brain electrical fields. Hum Brain Mapp $1: 134-152$.

Tucker DM, Luu P, Pribram KH (1995) Social and emotional selfregulation. Ann NY Acad Sci 769:213-239.

Tucker, DM, Hartry-Speiser A, McDougal L, Lure P, deGrandpre D (1999) Mood and spatial memory: emotion and the right hemisphere contribution to spatial cognition. Biol Psychol 50:103-125. 